\title{
The Interchange Instability in High-Latitude Plasma Blobs
}

\author{
P. K. Chaturvedi and J. D. Huba \\ Geophysical and Plasma Dynamics Branch, Plasma Physics Division \\ Naval Research Laboratory, Washington, D. C.
}

\begin{abstract}
The stability of high-latitude plasma density enhancements ("blobs") is analyzed with regard to the interchange mode (driven by neutral wind or equilibrium transverse electric field acting on the density gradient at the walls of the blobs). The effects arising from the finite parallel length of the blobs along the magnetic field lines are included in the analysis. Plasma regions of differing collisionalities, to which the blobs extend in altitude, are considered. We find that the finite parallel blob size results in a modest reduction in the growth rates of the small $(\$ 1 \mathrm{~km})$ and intermediate $(1-10 \mathrm{~km})$ scale sizes but severely reduces the growth rates for the large scale sizes $(>10 \mathrm{~km})$ for the observed parallel blob lengths $(\sim 300-600 \mathrm{~km})$. Further, it is found that the instability growth rates show a moderate reduction at higher altitudes (where ion-inertial effects may be dominant over the ion-neutral collisional effects). Thus the $\mathbf{E} \times \mathbf{B}$ instability is considered a plausible candidate for the scintillation-causing irregularities (1-10 $\mathrm{km}$ ) associated with the high-latitude blobs.
\end{abstract}

\section{INTRODUCTION}

It has long been known that the ionosphere at high latitudes often displays density irregularities and structured electric fields which are of various magnitudes in scale and strength [Fejer and Kelley, 1980]. The recent resurgence in interest in high-latitude irregularities has been due to the observation of scintillations by the Defense Nuclear Agency's Wideband Satellite in association with the gradient in the total electron content (TEC) at these latitudes [Fremouw et al., 1977; Rino et al., 1978]. A variety of experimental techniques have now been employed to study the structure at high latitudes, and its morphology is beginning to be understood better. On the basis of incoherent scatter radar returns, the plasma density contours have revealed the existence of highlatitude enhancements which have scale sizes transverse to and along the magnetic field of the order of hundreds of kilometers and have frequently been referred to as "blobs" [ Vickrey et al., 1980; Kelley et al., 1982]. Simultaneous incoherent scatter and VHF scintillation measurements were used by Vickrey et al. [1980] to establish the colocation of $\sim 1-\mathbf{k m}$ scale irregularities and the $F$ layer blobs. Ground-based optical and digital ionosonde measurements, in conjunction with the particle measurements on the DE 2 satellite, have found the presence of large-scale plasma irregularities (called "patches") at polar cap latitudes [Weber et al., 1984]. Various mechanisms have been proposed for the cause of these largescale plasma irregularities, such as generation by particle precipitation and convection from other regions [Kelley et al., 1982; Kelley and Vickrey, 1984; Weber et al., 1984; Sojka and Schunk, 1986]. Associated with the large-scale blobs, there are density gradients at "walls" of these blobs (primarily in the horizontal east-west direction) that are observed to be structured [Vickrey et al., 1980; Rino et al., 1983; Basu et al., 1984; Cerisier et al., 1985; Tsunoda et al., 1985]. It is generally believed that the structure at the so-called "intermediate" scale sizes $(\sim 1-10 \mathrm{~km})$ is responsible for the observed satellite scintillations.

This paper is not subject to U.S. copyright. Published in 1987 by the American Geophysical Union.

Paper number 6A8773.
A plasma blob at high latitudes is reminiscent of artificially released clouds in the ionosphere, and this fact has motivated us to do analogous analysis of the $\mathbf{E} \times \mathbf{B}$ instability for the blob situation. In this connection, we mention that the $\mathbf{E} \times \mathbf{B}$ instability has been applied to the naturally occurring transverse density gradients at mid-latitudes [Scannapieco et al., 1975] and to the high-latitude situation [Keskinen and Ossakow, 1982, 1983; Mitchell et al., 1985]. In addition, we note here that yet another gradient-driven instability, the current convective instability (which occurs in the presence of a parallel current), has been invoked to interpret high-latitude structure in situations which may be $\mathbf{E} \times \mathbf{B}$ stable [Ossakow and Chaturvedi, 1979; Chaturvedi and Ossakow, 1979; Huba and Ossakow, 1980; Chaturvedi and Ossakow, 1981]. Most of the past work for the $\mathbf{E} \times \mathbf{B}$ and current convective instability was carried out in the approximation of an infinitely long blob parallel to the magnetic field. These earlier studies clearly need to be modified for the blob-related structure, since, as has been noted earlier, the blobs have finite length in the direction parallel to the field lines. The work on the $\mathbf{E} \times \mathbf{B}$ instability in barium clouds has shown that the growth rate is severely modified for the long-wavelength modes when the finite parallel size of the cloud is considered [Völk and Haerendel, 1971; Goldman et al., 1976; Sperling et al., 1984; Drake et al., 1985; Drake and Huba, 1986]. A similar analysis carried out for the current convective instability has demonstrated that the instability growth rate is reduced of the order of 2 orders of magnitude for the scintillation-causing scale sizes (for the observed blob sizes) [Huba and Chaturvedi, 1986].

In this paper we investigate the $\mathbf{E} \times \mathbf{B}$ instability for the high-latitude bloblike situation. Thus in our analysis we have included the effects of the finite parallel blob size, and ion inertia (which can become important at high altitudes where the ion-neutral collision frequency is small [Ossakow et al., 1978]). We find that the growth rate for the $\mathbf{E} \times \mathbf{B}$ instability is modified by the finite parallel size of the blob, with the longer wavelength showing more severe reductions in growth rate than the small and intermediate scale sizes. The effect of ion inertia at high altitudes also leads to a reduction in the growth rate, but the reduction is relatively moderate for the small and intermediate wavelengths $(\lambda \lesssim 10 \mathrm{~km})$ but is substantial at the long wavelengths $(\lambda \gtrsim 10 \mathrm{~km})$. Further, the mode is localized in the blob region parallel to the magnetic 
field. This has some notable consequences. For instance, the mode may remain relatively unaffected by the regions afar. Thus the blob-associated structure of $F$ region altitudes may develop even in the presence of a conducting $E$ region. The mapping of structure-associated potential fields is also modified because of the localization. The electrostatic potential is roughly constant within the enhancement (cloud), but as it maps into the background ionosphere, the mapping distance is finite because of the dissipation. This mapping, originally discussed by Farley [1959], follows the relationship $\left(L_{||} / L_{\perp}\right) \propto\left(\sigma_{\|} / \sigma_{\perp}\right)^{1 / 2}$, where $L$ is the characteristic field scale size, $\sigma$ is the conductivity of the medium, and the subscripts $\|$, $\perp$ refer to the parallel and transverse components to the magnetic field, respectively. We find that this mapping may not extend to large parallel distances for structured fields at higher altitudes where $v_{\text {in }}$ (and thus $\sigma_{\perp}$ ) is very small. We discuss these results in the final section.

The plan of the paper is as follows. In the next section we present the basic equations and briefly discuss the equilibrium. In the third section the nonlocal mode equation is derived. The fourth section contains the numerical results. Finally, in the fifth section the results are discussed.

\section{Basic Equations and Equilibrium}

A set of nonlinear equations describing the evolution of a plasma density enhancement has been derived by Sperling et al. [1984] and Drake et al. [1985]. We briefly present the derivation here. A plasma blob is assumed to have finite length along the uniform magnetic field $B=B_{0} \hat{z}$ with a background neutral wind $V_{n}=V_{n} \hat{x}$. The plasma is assumed to be cold (i.e., $T=0$ ); see Appendix $A$ for a discussion of finite temperature effects. The basic equations are

$$
\begin{gathered}
\frac{\partial n}{\partial t}+\nabla \cdot\left(n \mathbf{v}_{e}\right)=0 \\
\mathbf{0}=-e \mathbf{E}-\frac{e}{c} \mathbf{v}_{e} \times \mathbf{B}-m_{e} v_{e n}\left(\mathbf{v}_{e}-\mathbf{v}_{n}\right)-m_{e} v_{e i}\left(\mathbf{v}_{e}-\mathbf{v}_{i}\right) \\
m_{1}\left(\frac{\partial}{\partial t}+\mathbf{v}_{i} \cdot \nabla\right) \mathbf{v}_{i}=e \mathbf{E}+\frac{e}{c} \mathbf{v}_{i} \times \mathbf{B} \\
-m_{i} v_{i n}\left(\mathbf{v}_{i}-\mathbf{v}_{n}\right)-m_{i} v_{i e}\left(\mathbf{v}_{i}-\mathbf{v}_{e}\right) \\
\nabla \cdot \mathbf{J}=\nabla \cdot\left[n e\left(\mathbf{v}_{i}-\mathbf{v}_{e}\right)\right]=0 \\
\nabla \times \mathbf{B}=\frac{4 \pi}{c} \mathbf{J}
\end{gathered}
$$

In the above, $n_{\alpha}$ and $v_{\alpha}$ are the density and fluid velocity, respectively, of species $\alpha, v_{a n}$ is the $\alpha$ species-neutral collision frequency, $v_{e i}$ is the electron-ion collision frequency, $v_{i e}$ is the ion-electron collision frequency, and $\alpha$ refers to electrons $(e)$ or ions (i). The electric and magnetic fields are represented in terms of potentials as

$$
\begin{aligned}
& \mathbf{E}=-\nabla \phi-\frac{1}{c} \frac{\partial A_{z}}{\partial t} \hat{z} \\
& \mathbf{B}=B_{0} \hat{z}+\nabla A_{z} \times \hat{z}
\end{aligned}
$$

where $\phi$ is the electrostatic potential and $A_{z}$ is the vector potential associated with the magnetic field produced by the self-consistent plasma currents. We consider only $A_{z}$ since $J_{\|} \gg J_{\perp}$ and assume that $\left|\nabla A_{z} \times \hat{z}\right| \ll B_{0}$.

The electron motion transverse and parallel to $B_{0}$ is de- scribed as

$$
\mathbf{v}_{e \perp}=-\frac{c}{B} \nabla_{\perp} \phi \times \hat{z}
$$

and

$$
v_{\| e}=-e E_{\|} /\left[m_{e}\left(v_{e}+f v_{e i}\right)\right]
$$

with

$$
E_{\|}=-\nabla_{\|} \phi-\frac{1}{c} \frac{\partial A_{z}}{\partial t}
$$

where $v_{e}=v_{e i}+v_{e n}$ and $\nabla_{\|}=b \cdot \nabla, b=\mathbf{B} /\left|\mathbf{B}_{0}\right|$. Also, with $v_{e} / \Omega_{e} \ll 1$ and $v_{i n} \lesssim \Omega_{i}, f \equiv m_{e} v_{e n} / m_{i} v_{i n} \ll 1$. The ion dynamics is given by

$$
\begin{gathered}
\mathbf{v}_{1 \perp}=\varepsilon_{i}\left[-\frac{c}{B} \nabla_{\perp} \phi \times \hat{z}+\frac{\tilde{v}_{i n}}{\Omega_{\imath}} \mathbf{V}_{n} \times \hat{z}\right. \\
\left.\quad-\frac{\tilde{v}_{\text {tn }}}{\Omega_{i}} \frac{c}{B} \nabla_{\perp} \phi+\left(\frac{\tilde{v}_{i n}}{\Omega_{i}}\right)^{2} \mathbf{V}_{n}\right] \\
v_{\| i}=e E_{\|} v_{e n} /\left[m_{i} \tilde{v}_{i n}\left(v_{e}+f v_{e i}\right)\right]
\end{gathered}
$$

where $\varepsilon_{i}=\left(1+v_{i n}{ }^{2} / \Omega_{i}{ }^{2}\right)^{-1}$ and $\tilde{v}_{i n}=v_{i n}+\partial / \partial t$. In (11), both Pedersen and Hall responses to the electric field and neutral wind have been included. Note also that we have included the ion inertia $\left(\partial / \partial t\right.$ in $\left.\tilde{v}_{i n}\right)$ in the above, even though the full convective derivative has not been included (the $v \cdot \nabla v$ term is not taken into account). The reason for doing so is the fact that this term only introduces a real frequency to the solution and does not affect the stability criterion for the wavelengths of interest.

We present the equations derived by Sperling et al. [1984] for the $\mathbf{E} \times \mathbf{B}$ instability with the ion-inertial effects included These equations, derived from (8) (12) and (1), (4), and (5), are

$$
\begin{gathered}
\frac{d n}{d t}-\frac{c}{B} \nabla \hat{\phi} \times \hat{z} \cdot \nabla n+\nabla_{\|} \frac{c}{4 \pi e} \nabla_{\perp}{ }^{2} A_{z}=0 \\
\varepsilon_{i} \frac{c}{B} \frac{\tilde{v}_{i n}}{\Omega_{i}} \nabla_{\perp} \cdot n \nabla_{\perp} \hat{\phi}+\varepsilon_{\mathrm{i}} \frac{c}{B} \frac{\tilde{v}_{t m}{ }^{2}}{\Omega_{\imath}{ }^{2}} \hat{z} \times \nabla \hat{\phi} \cdot \nabla n \\
+\frac{\tilde{v}_{t n}}{\Omega_{i}} \hat{z} \times \mathbf{V}_{n} \cdot \nabla n+\frac{c}{4 \pi e} \nabla_{\|} \nabla_{\perp}{ }^{2} A_{z}=0 \\
\nabla_{\perp}{ }^{2} A_{z}=\frac{4 \pi}{c \eta_{e}}\left(\nabla_{\|} \phi+\frac{1}{c} \frac{d A_{z}}{d t}\right)
\end{gathered}
$$

where $\eta_{e}=m_{e} v_{e} / n e^{2}$ is the parallel resistivity and

$$
\begin{gathered}
\phi \equiv \phi-\frac{B}{c} \frac{\tilde{v}_{i n}}{\Omega_{i}} \mathbf{V}_{n} \cdot \hat{x} \\
\frac{d}{d t} \equiv \frac{\partial}{\partial t}+\frac{v_{t n}}{\Omega_{\imath}} \hat{z} \times V_{n} \cdot \nabla \\
\nabla_{\|}=\frac{\partial}{\partial z}+B_{0}{ }^{-1} \nabla A_{z} \times \hat{z} \cdot \nabla
\end{gathered}
$$

Equation (13) is the electron continuity equation, (14) is the charge conservation equation, and (15) is Ampere's law. Now we shall discuss the equilibrium of these equations briefly.

The equilibrium of a cold plasma enhancement has been discussed by Sperling et al. [1984]. A two-dimensional cloud localized along and across the magnetic field $\mathbf{B}_{0}, n_{t}=n_{i}(x, z)$ 
with $n_{i} \neq 0$ for $|x| \leq x_{0}$ and $|z| \leqslant z_{0}$ is described by

$$
\begin{gathered}
\frac{\partial n}{\partial t}+\frac{c}{4 \pi e} \frac{\partial}{\partial z} \frac{\partial^{2} A_{z}}{\partial x^{2}}=0 \\
\varepsilon_{i} \frac{c}{B} \frac{v_{i n}}{\Omega_{i}} \frac{\partial}{\partial x} n \frac{\partial}{\partial x} \hat{\phi}+\frac{c}{4 \pi e} \frac{\partial}{\partial z} \frac{\partial^{2} A_{z}}{\partial x^{2}}=0 \\
\frac{\partial^{2} A_{z}}{\partial x^{2}}=\frac{4 \pi}{c \eta_{e}}\left(\frac{\partial \hat{\phi}}{\partial z}+\frac{1}{c} \frac{\partial A_{z}}{\partial t}\right)
\end{gathered}
$$

In the above, it has been assumed that the background ionosphere is uniform throughout the region, and $x_{0}$ and $z_{0}$ are the typical transverse and longitudinal scales, respectively, of the plasma enhancements. From (19)-(21) we find that the equilibrium is given by $A_{z}=\delta=0$ with $n(x, z)$ an arbitrary function. For simplicity we consider the plasma density to be given by

$$
\begin{array}{llrl}
n(x, z) & =n_{1} & z & <-z_{0} \\
n(x, z) & =n_{i}(x) & -z_{0} & <z<z_{0} \\
n(x, z) & =n_{2} & z & >z_{0}
\end{array}
$$

\section{Linear Analysis and Dispersion Relation}

We perturb the set of equations (13)-(15) over the equilibrium discussed in the previous section and linearize the equations with respect to perturbations. The perturbed quantities are assumed to vary as $f \sim f(z) \exp \left(\gamma t+k_{y} y\right)$. The above set then leads to

$$
\begin{gathered}
\left(k_{y}{ }^{2} D_{r}+\gamma\right) \tilde{A}_{z} \simeq-c \frac{\partial \tilde{\phi}}{\partial z} \\
{\left[\gamma\left(1+\frac{\gamma}{v_{i n}}\right)-\gamma_{0}\right] \tilde{\phi} \simeq-\frac{\gamma \alpha D_{r}}{c} \frac{\partial \tilde{A}_{z}}{\partial z}}
\end{gathered}
$$

The above equations are the same as those discussed in the work of Sperling et al. [1984] with the exception of the second term in parentheses on the left-hand side of $(24)\left(\gamma / v_{i n}\right)$ which represents the effects of ion inertia. The following definitions have been used in (23) and (24): $\gamma_{0}=V_{n} / L_{n}, L_{n}=$ $\left(\partial \ln n_{i} / \partial x\right)^{-1}, D_{r}=v_{e i} c^{2} / \omega_{p e}{ }^{2}, \omega_{p e}{ }^{2}=4 \pi n_{0} e^{2} / m_{e}, \alpha=\Omega_{e} \Omega_{i} /$ $v_{e i} v_{i n}$, and $v_{i n} / \Omega_{i} \ll 1$ was assumed. We discuss some wellknown results from (23) and (24) in the local analysis in Appendix A. Now we present a derivation of the nonlocal dispersion relation.

The method of solving (23) and (24) we follow here is similar to that used by Sperling et al. [1984], Drake et al. [1985], and Huba and Chaturvedi [1986]. In the outer regions (outside the plasma blob) the ambient ionosphere is assumed to have no density gradient, and a decaying solution is assumed for the modes in these regions. We shall distinguish between the two outside regions by their differing ambient parameters (density, $v_{i n}$, etc.). We refer to the region below the blob as region 1 (bottomside) and the one above it as the region 2 (topside). In these regions the solution is written as

$$
\begin{array}{lll}
\tilde{\phi}=\tilde{\phi}_{1} e^{k_{1} z} & \tilde{A}_{z}=\tilde{A}_{1} e^{k_{1} z} & z<-z_{0} \\
\tilde{\phi}=\tilde{\phi}_{2} e^{-k_{2} z} & \tilde{A}_{z}=\tilde{A}_{2} e^{-k_{2} z} & z>z_{0}
\end{array}
$$

The inverse of decay lengths of the mode-associated fields in the ambient ionosphere is given by

$$
\frac{k_{p}^{2}}{k_{y}^{2}}=\left[\frac{\left[1+\left(\gamma / v_{i n}\right)\right]}{\alpha R}\right]_{p} \quad p=1,2
$$

where

$$
R=\frac{k_{y}^{2} D_{r}}{\gamma+k_{y}^{2} D_{r}}
$$

In the above, $p=1,2$ refer to the two outer regions as noted earlier, the term $\left(\gamma / v_{t n}\right)$ in (26) describes the effects of ion inertia, and $R$ is a measure of the electromagnetic effects. For the eletrostatic case $\left(k_{y}{ }^{2} D_{r} \gg \gamma\right), R \approx 1$, and for the electromagnetic case $\left(\gamma \gg k_{y}{ }^{2} D_{r}\right), R \simeq k_{y}{ }^{2} D_{r} / \gamma \ll 1$.

Inside the blob the mode is represented by a plane wave solution,

$$
\bar{\phi}=\tilde{\phi}_{i} e^{i k_{i} z} \quad \tilde{A}_{z}=\tilde{A}_{z i} e^{i k_{i z}} \quad-z_{0}<z<z_{0}
$$

with the parallel wave number of the mode inside the blob being given by

$$
\frac{k_{i}^{2}}{k_{y}^{2}}=\left\{\frac{\left\{\gamma_{0}-\gamma\left[1+\left(\gamma / v_{i n}\right)\right]\right\}}{\gamma} \frac{1}{\alpha R}\right\}_{i}
$$

where the quantities are evaluated in the blob region and the ion-inertial effects are represented by the third term in the numerator of (29). A solution is constructed from (29) for the mode, using the two roots of $k_{i}$ ( $k_{t \pm}$ corresponding to the positive and negative roots),

$$
\tilde{A}_{i}=\widehat{A} e^{i k_{i+z}}+\hat{B} e^{i k_{1-z}}
$$

A similar solution is written out for $\tilde{\phi}_{i}$. The solutions are matched at the boundaries of the inner and outer regions to derive the dispersion relation. The appropriate matching conditions are obtained by integrating (23) and (24) across the boundaries $\left(z= \pm z_{0}\right)$; it is found that $\tilde{\phi}$ and $\tilde{A}_{z}$ are continuous at $z= \pm z_{0}$. Using (25) and (30) in (23) and (24) with these matching conditions, we find the dispersion equation is

$$
k_{i} z_{0}=\frac{1}{2} \tan ^{-1}\left(\frac{k_{1}}{k_{i}}+\frac{k_{2}}{k_{i}}\right)\left(1-\frac{k_{1} k_{2}}{k_{i}^{2}}\right)^{-1}+m \pi
$$

where $m$ is an integer. The largest growth rate occurs for the $m=\mathbf{0}$ mode, and we consider only this mode henceforth. The wave numbers $k_{1,2}$ and $k_{i}$ are defined in (26) and (29), respectively. Equation (31) is solved numerically, but first we shall discuss some simple analytical results from it.

The results of local analysis can be obtained from (31) in the limit $(1-\hat{\gamma}) / \hat{\gamma} \ll 1$, where $\hat{\gamma}=\gamma / \gamma_{0}$, in which case one may write $\tan \left(2 k_{i} z_{0}\right) \simeq 2 k_{i} z_{0}$ and the dispersion relation is

$$
2 k_{i} z_{0} \simeq \pi / 2
$$

By identifying $k_{z e f f}{ }^{2}=\pi^{2} / 16 z_{0}{ }^{2}$, one is able to recover the local theory results presented in Appendix A from (32).

The finite length of the blob and the ion inertia both contribute to a reduction of the $\mathbf{E} \times \mathbf{B}$ instability growth rate. We have noted earlier that we distinguish the ionospheric regions above and below the blob by their differing ambient parameters. We shall also distinguish three separate cases in which the effects of ion inertia are introduced differently (see Tables 1 and 2). In case I, both the topside and the bottomside ionosphere, as well as the blob, correspond to ionospheric altitudes where the ion-inertial effects are neglected. In case II the ioninertial effects are introduced only in the topside ionosphere region, and in case III, both the blob and the topside ionosphere are considered at altitudes where ion-inertial effects may become important. We present some simple analytical expressions for the growth rate of the $\mathbf{E} \times \mathbf{B}$ instability for the 
TABLE 1. Ionospheric Parameters

\begin{tabular}{|c|c|c|c|c|c|c|c|}
\hline & \multirow[b]{2}{*}{ Bottomside* } & \multicolumn{2}{|c|}{ Case I } & \multicolumn{2}{|c|}{ Case II } & \multicolumn{2}{|c|}{ Case III } \\
\hline & & Blob & Topside & Blob & Topside & Blob & Topside \\
\hline $\begin{array}{l}\text { Plasma density, } \mathrm{cm}^{-3}(n) \\
\text { Electron-ion collision }\end{array}$ & $\begin{array}{l}5 \times 10^{5} \\
10^{3}\end{array}$ & $10^{4} \times 10^{6}$ & $\begin{array}{l}10^{6} \\
5 \times 10^{3}\end{array}$ & $10^{4} \times 10^{6}$ & $\underset{10^{3}}{5 \times 10^{5}}$ & $\underset{10^{4}}{5 \times 10^{6}}$ & $\stackrel{10^{5}}{5 \times 10^{2}}$ \\
\hline $\begin{array}{l}\text { frequency }\left(v_{e t}\right) \\
\text { Plasma frequency }\left(\omega_{p e}\right) \\
\text { Ion-neutral collision }\end{array}$ & $\begin{array}{l}3.78 \times 10^{7} \\
30\end{array}$ & $\begin{array}{l}1.26 \times 10^{8} \\
0.5\end{array}$ & $\begin{array}{l}5.64 \times 10^{7} \\
0.05\end{array}$ & $\begin{array}{l}1.26 \times 10^{8} \\
0.1\end{array}$ & $\begin{array}{l}3.78 \times 10^{7} \\
0.01\end{array}$ & $\begin{array}{l}1.26 \times 10^{8} \\
0.05\end{array}$ & $\begin{array}{l}1.78 \times 10^{7} \\
0.01\end{array}$ \\
\hline $\begin{array}{l}\text { frequency }\left(v_{t n}\right) \\
\alpha=\left(\Omega_{e} \Omega_{i} / v_{e l} v_{t n}\right) \\
D_{r}=v_{e} c^{2} / \omega_{p e}{ }^{2} \\
L=(D)\end{array}$ & $10^{5}$ & $\begin{array}{l}6 \times 10^{5} \\
5.625 \times 10^{8} \\
2.37 \times 10^{5}\end{array}$ & $1.2 \times 10^{7}$ & $3 \times 10^{6}$ & $3.10^{8}$ & $6 \times 10^{6}$ & $6 \times 10^{8}$ \\
\hline $\begin{array}{l}L_{r}=\left(D_{r} / \gamma_{0}\right) \\
z_{r}=\alpha^{1 / 2} L_{r} \\
\hat{z_{0}}=z_{0} / z_{r}\end{array}$ & $\begin{array}{l}1.84 \times 10^{8} \\
0.16\end{array}$ & & $\begin{array}{l}4.1 \times 10^{8} \\
0.07\end{array}$ & & $\begin{array}{l}5.8 \times 10^{8} \\
0.05\end{array}$ & & \\
\hline
\end{tabular}

Some common parameters that have been used are $T_{e}=T_{i}=0.1 \mathrm{eV} ; B_{0}=0.5 \mathrm{G} ; \Omega_{e}=10^{7}, \Omega_{i}=3 \times 10^{2} ; L_{N}=4 \times 10^{6} \mathrm{~cm} ; \gamma_{0}=V_{n} / L_{n}=$ $10^{-2} \mathrm{~s}^{-1} ; 2 z_{0}=300 \mathrm{~km}$.

* Bottomside parameters have been chosen to be the same for all three cases.

above three cases in Appendix B. It is shown there that the growth rate is reduced for a finite length blob and the reduction is more pronounced at the long transverse wavelengths. Also, the earlier results of Sperling et al. [1984] are recovered for the case when regions 1 and 2 may be approximated in the collisional limit with the same parameters. We shall further discuss the results for the three cases in the discussion section along with the numerical results which will be presented next.

\section{Numerical Results}

We have solved the nonlocal dispersion relation (31) for parameters appropriate to the high-latitude blob situation in three different cases as described in the previous section. The parameters used have been tabulated in Tables 1 and 2 . We use the following normalizations: $\hat{\gamma}=\gamma / \gamma_{0}, \hat{k}_{y}=k_{y} L_{r}, \hat{z}_{0}=$ $z_{0} / z_{r}, z_{r}^{2}=\alpha_{i} L_{r i}^{2}$, and $L_{r}^{2}=D_{r} / \gamma_{0}$. The blob length, $2 z_{0}$, has been assumed to be the same in the three cases and is taken to be $300 \mathrm{~km}$. Similarly, the gradient scale length $L_{N}$ associated with the "walls" of the blob is taken to be $40 \mathrm{~km}$, and the neutral wind velocity $V_{n}$ is taken to be $400 \mathrm{~m} / \mathrm{s}$ in all three cases, resulting in the linear local theory growth rate $\gamma_{0} \sim$ $10^{-2} \mathrm{~s}^{-1}$. Most of the other parameters and symbols are explained in Tables 1 and 2 and will be stated when introduced below. In the following the growth rate expressions have been computed as a function of wave number, the blob length, and the ion collision frequency for various cases. In addition, we have also plotted the wave function within and outside the blob for various cases, to display the localization of the mode around the instability region (i.e., the blob). For reference we note that $1-\mathrm{km}$ mode correspond to $k_{y} L_{r}=14.5$ and $10-\mathrm{km}$ modes correspond to $k_{y} L_{r}=1.45$.

Figure 1 shows the normalized growth rate $\gamma / \gamma_{0}$ plotted as a function of normalized transverse wave number $k_{y} L_{r}$ for the three cases mentioned above. In case I (curve A), the bottomside, blob, and topside are all taken to be collisional, and ion-inertial effects are unimportant. For large $k_{y}$ (short transverse wavelengths) the growth rate can be seen to approach asymptotically the local theory growth rate, $\gamma \simeq \gamma_{0}$. For small $k_{y}$ (long transverse wavelengths) the growth rate is reduced. The intermediate scale size regime corresponding to $1-10 \mathrm{~km}$ is of special interest, for these irregularities are associated with the signal scintillations. It can be seen that the finite blob length has little effect on the 1-km scale size mode, where growth rate is close to the local theory value $\left(\gamma \simeq 0.9 \gamma_{0}\right)$, but at the 10-km scale size end, the growth rate is down by almost an order of magnitude $\left(\gamma \simeq 0.2 \gamma_{0}\right)$. When inertial effects are introduced for the topside (case II, curve $B$ ), the growth rate curve shows an overall decrease, and the reduction at long wavelengths becomes more pronounced. Thus the $1-\mathrm{km}$ end of intermediate scale size shows a small reduction in growth rate $\left(\gamma \simeq 0.75 \gamma_{0}\right)$, while the $10-\mathbf{k m}$-wavelength modes suffer severe reduction $\left(\gamma \simeq 0.05 \gamma_{0}\right)$ in growth rate. This trend continues for the case when the inertial effects are important for both the blob and the topside (case III, curve C). Now even the local growth rate (in the inertial limit) is different from the value in the collisional case $\left(\gamma \simeq 0.6 \gamma_{0}\right.$ in the inertial case, while $\gamma \simeq \gamma_{0}$ in the collisional case). The 1-km scales show less reduction in the growth rate due to the finite blob length effects $(\hat{\gamma} \simeq$ $0.35 \gamma_{0}$ ) than the $10-\mathrm{km}$ size scales $\left(\gamma \simeq 0.025 \gamma_{0}\right)$, as expected.

In Figures $2 a$ and $2 b$ the explicit effect of finite blob size is shown on the growth rates of $1-\mathrm{km}$ and $10-\mathrm{km}$ size modes for cases I and III, respectively. Figure $2 a$ refers to case I, and we see that the $1-\mathrm{km}$ scale sizes (curve A) experience lesser reductions in the growth rate than the $10-\mathrm{km}$ size modes (curve B). It can be seen that for very long blobs (approaching the infinitely long blob limit, or the local theory limit) the growth rate for $k_{y} L_{r} \simeq 14.5\left(\lambda_{\perp} \sim 1 \mathrm{~km}\right)$ approaches the local theory value $\left(\gamma \simeq \gamma_{0}\right.$ ) and for small blob lengths the growth rate is progressively reduced. This pattern is more pronounced for the longer wavelengths $\left(k_{y} L_{r} \simeq 14.5, \lambda_{\perp} \sim 10 \mathrm{~km}\right)$ for which the growth rate in the infinite blob limit $\left(\gamma \simeq 0.8 \gamma_{0}\right)$ is less than the local theory value, $\gamma \simeq \gamma_{0}$ (which is valid only for short wavelengths such that $k_{y} L_{N} \gg 1$ ). A similar trend is found for growth rate behavior as a function of blob length, when the inertial effects are introduced. This is shown in Figure $2 b$, and

TABLE 2. Parameters Used in Numerical Computation

\begin{tabular}{lccc}
\hline & Case I & Case II & Case III \\
\hline$\alpha_{1} / \alpha_{1}$ & 6 & 30 & 60 \\
$\alpha_{i} / \alpha_{2}$ & 0.05 & 0.01 & 0.01 \\
$\bar{v}_{i 1}=v_{i n}{ }^{(1)} / \gamma_{0}$ & 3000 & 3000 & 3000 \\
$\bar{v}_{1}{ }^{(i)}=v_{i n}{ }^{(1)} / \gamma_{0}$ & 50 & 10 & 5 \\
$\bar{v}_{i 2}=v_{i n}{ }^{(2)} / \gamma_{0}$ & 5 & 1 & $0.1^{*}$ \\
$\hat{z}_{0}$ & 0.2 & 0.1 & 0.05 \\
\hline
\end{tabular}

*Notice that $\alpha_{2}$ was computed using the value $\bar{v}_{\text {in2 }}=1$. 


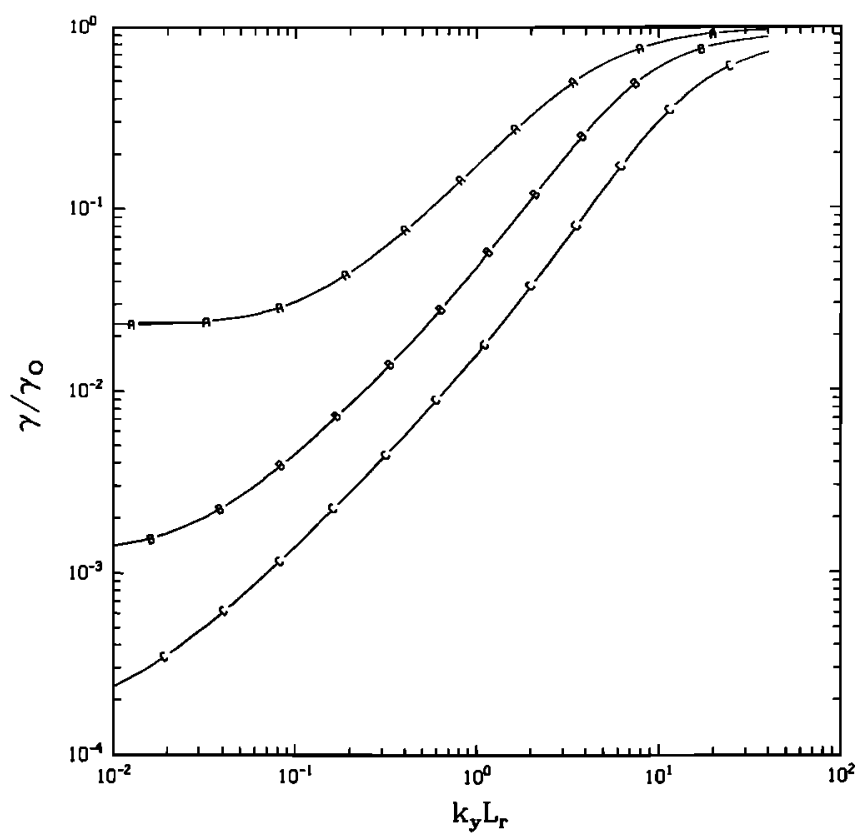

Fig. 1. Plot of the normalized growth rate $\gamma / \gamma_{0}$ versus normalized transverse wave number $k_{y} L_{r}$ for the parameters listed in Table 2. Curve A corresponds to case $\mathbf{I}$, curve $B$ to case II, and curve $C$ to case III.

the inertial effects have been included for both the blob and the topside regions. The growth rates for both the scale sizes $(\lambda \sim 1,10 \mathrm{~km})$ are reduced in this (inertial) case for the infinite blob limit from the value corresponding to the collisional limit. And as the blob lengths get smaller, the reduction in the growth rate is more, with longer wavelengths (curve B) showing more pronounced reduction than the small wavelengths (curve A).

In Figures 3-5 we have plotted the wave functions for the modes within the blob (the instability region) and outside it (the ambient ionosphere) to show the localization of the modes in the blob region and its extension into the ambient ionosphere. In Figure 3 the wave function is plotted for the short wavelength mode $\left(k_{y} L_{r} \simeq 14.5, \lambda_{\perp} \sim 1 \mathrm{~km}\right)$. Figures $3 a$ and $3 b$ refer to cases I and III, respectively. It can be easily seen that in the collisional bottomside region the mode extends to smaller distances in the ambient ionosphere than the topside ionosphere where collisions are low. This mapping, dependent on the ambient collisionality, shows for the collisional topside (Figure $3 a$ ) the mode extending to smaller distances than the case in which the topside is assumed inertial (Figure $3 b$ ).

The mapping of modes into the ambient ionosphere is also scale size dependent. This is shown in Figures 4 and 5 . In Figure 4, wave numbers $k_{y} L_{r} \simeq 14.5$ (Figure $4 a$ ) and $k_{y} L_{r} \simeq$ 1.45 (Figure $4 b$ ) are plotted for the case when all the regions are taken to be collisional (case I, Table 2). Clearly, the longer wavelengths extend farther into the background than the smaller wavelengths. In Figure 5, which corresponds to the case in which the topside is assumed inertial (case III, Table 2), this pattern is repeated, with the potential fields mapping to larger distances on the topside (which is less collisional) than on the bottomside (which is more collisional). Again, as one would expect, this mapping is to larger distances for the longer-wavelength modes (Figures $5 b, k_{y} L_{r} \simeq 1.45$ ) than for the small-wavelength mode (Figure $5 a, k_{y} L_{r} \simeq 14.5$ ).

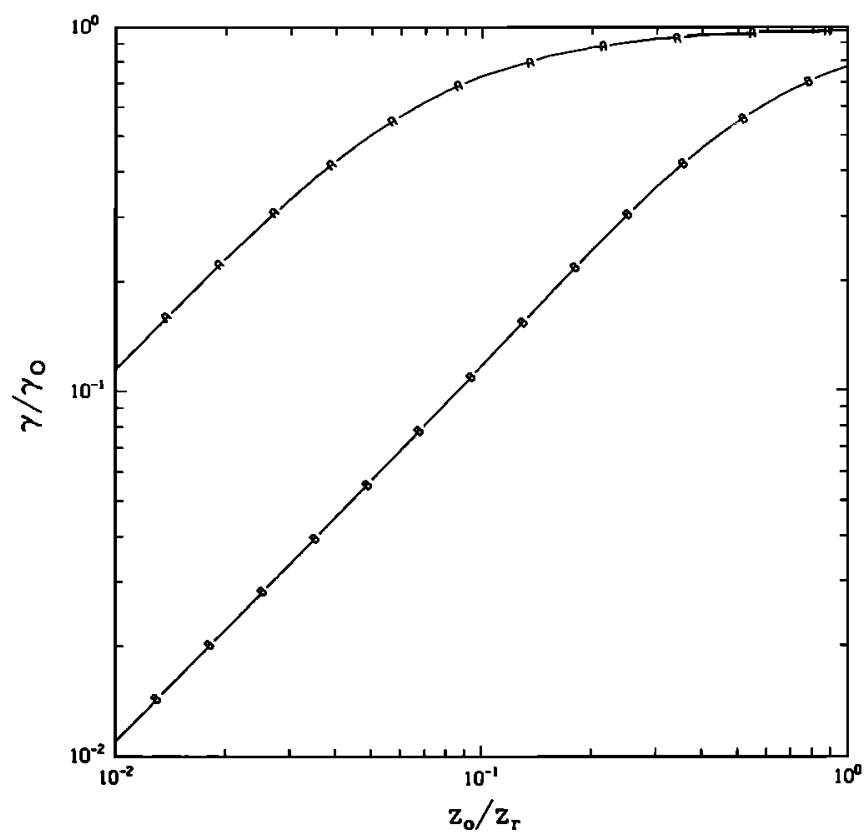

Fig. $2 a$

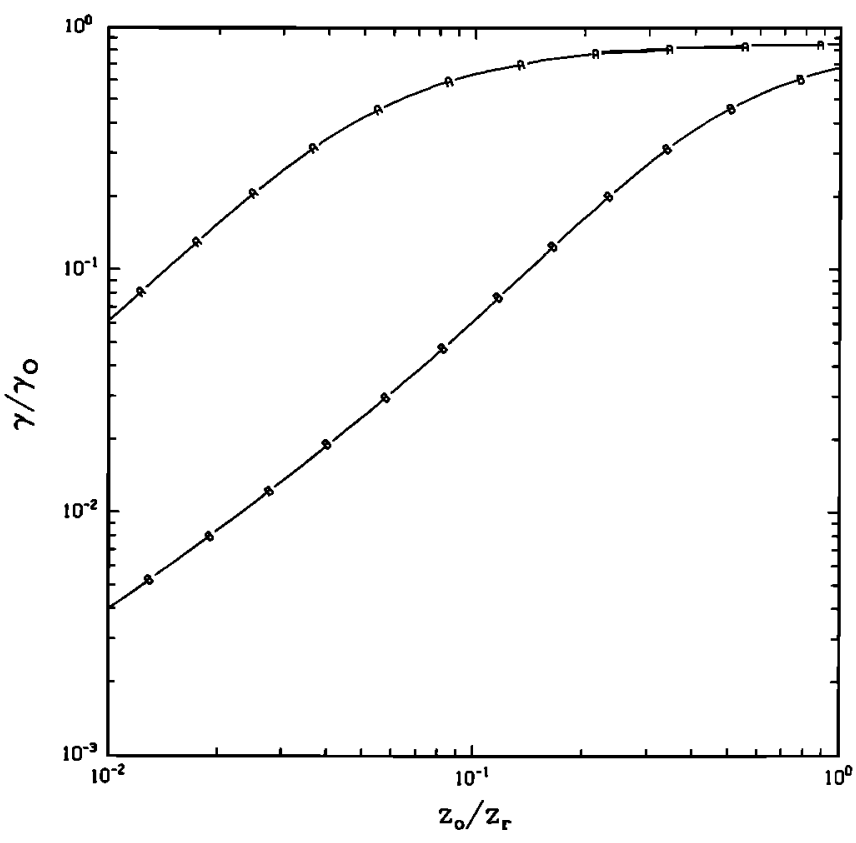

Fig. $2 b$

Fig. 2. Plot of the normalized growth rate $\gamma / \gamma_{0}$ versus normalized parallel blob length $z_{0} / z_{r}$. Curve A corresponds to $k_{y} L_{r}=14.5$, and curve $B$ corresponds to $k_{v} L_{r}=1.45$. (a) Parameters used are for case 1 . (b) Parameters used are for case III.

An interesting point to note from Figures $3-5$ is that in the highly collisional bottomside region the smaller scales do not map to large distances. Thus we see that the $1-\mathrm{km}$ size mode maps to less than the blob length, $z_{0} / 2(\sim 75 \mathrm{~km})$ in the collisional bottomside (Figures $3 a$ and $4 a$ ). This implies that this mode, depending on the altitude of the blob, may not be affected by a conducting $E$ region. A conducting $E$ region is, however, likely to affect the development of longer wavelengths ( $\gtrsim 10 \mathrm{~km}$ ), as is evident from Figures $3 b$ and $4 b$, where the modes extend to several times the blob length ( $\gtrsim 400 \mathrm{~km}$ ). 


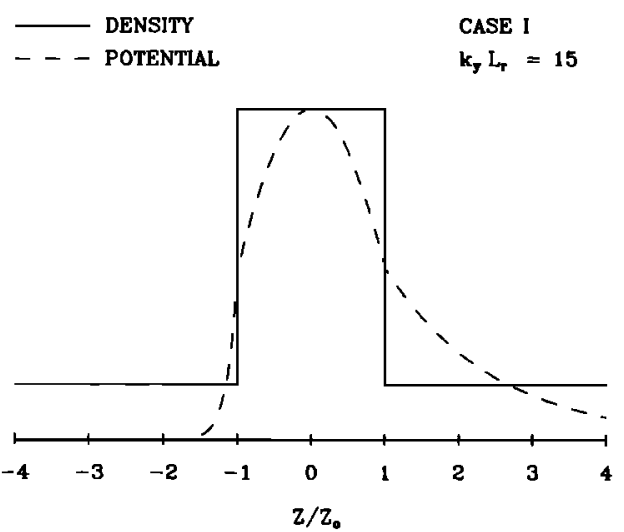

(a)
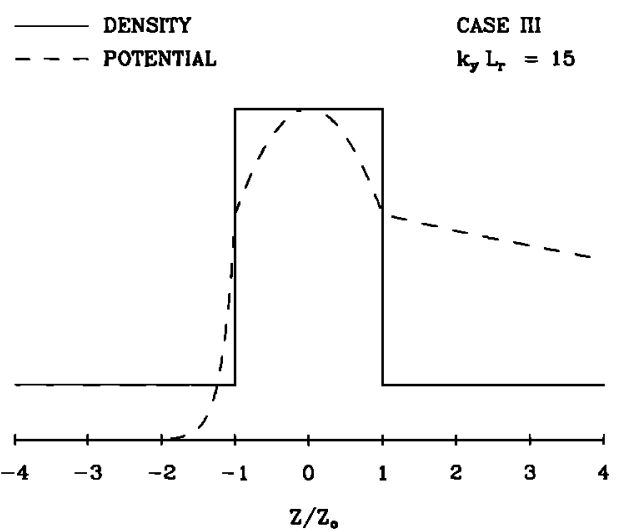

(b)

Fig. 3. Plot of density (solid line) and potential (dashed line) profiles as a function of blob length $\left(z_{0} / z_{r}\right)$. (a) Parameters used are for case I with $k_{y} L_{r}=14.5$. (b) Parameters used are for case III with $k_{y} L_{r}=14.5$.

\section{Discussion}

We have studied the effects of finite blob length on the interchange instability applicable to high latitudes and have included the effects of finite ion inertia. The experimental observations indicate that the high-latitude blobs are of finite extent parallel to the magnetic field with typical scale sizes of a few hundreds of kilometers [Tsunoda et al., 1985]. This means that they span regions of varying plasma parameters in altitude, e.g., the collision frequencies and density. At high altitudes the ion-neutral collision frequency $v_{\text {in }}$ decreases, and therefore the ion-inertial effects need to be included for the interchange instability analysis [Ossakow et al., 1978]. The introduction of finite parallel blob size in the analysis means that one should consider the finite parallel wavelength effects $\left(k_{\|} \neq 0\right)$ on the development of the instability, and it further implies that the coupling of the blob region to the ambient ionospheric regions should be taken into account.

There are some obvious similarities of dynamics between an artificial plasma cloud and a plasma blob in the ionosphere. Both represent a plasma density enhancement above the background, both are of finite dimensions, and in both cases their motion with respect to the background results in a steepened backside or a wall gradient which is observed to be striated or structured. Considering these common features, we have applied the theory of interchange instability developed for plasma clouds by Sperling et al. [1984] and Drake et al. [1985] to the blob situation, and we have included the ioninertial effects in the analysis. It may be pointed out that there are also some differences between the two situations. The clouds are relatively small in parallel size (tens of kilometers) as compared with the blob sizes (a few hundred kilometers) and have a sharper transverse density gradient. The constituent ions of clouds are also different (usually barium) from the background ions, while in the case of the blobs, the two are the same. Because of the large sizes, blobs occupy regions of diverse ionospheric properties in altitude. Thus the plasma properties at the bottom and the top of a blob may be very different in terms of collisionality, temperature, density, etc. This also has an important implication for the profile of the blob (as opposed to that of a cloud in whose case this size is quite small, and thus the properties of the two ionospheric regions adjacent to the cloud are not as different).

The analytical and numerical work on the stability of blobs

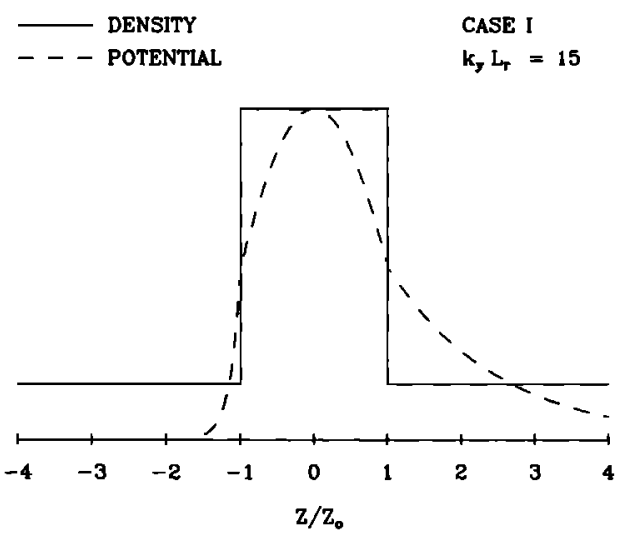

(a)

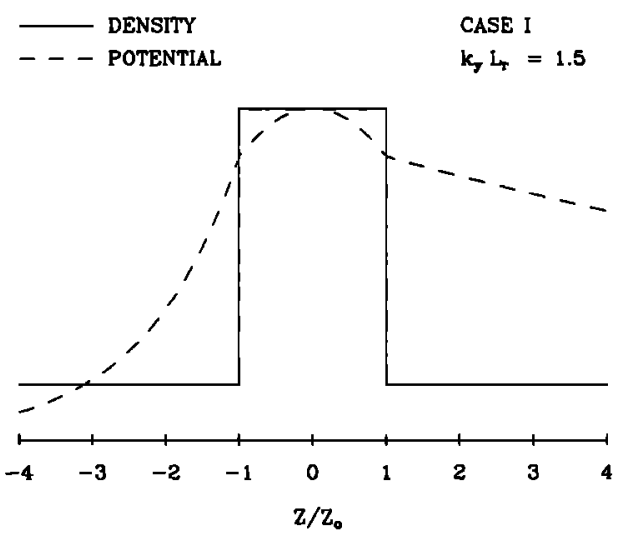

(b)

Fig. 4. Plot of density (solid line) and potential (dashed line) profiles as a function of blob length $\left(z_{0} / z_{r}\right)$. The parameters used are for case I: $(a) k_{y} L_{r}=14.5 ;(b) k_{y} L_{r}=1.45$. 


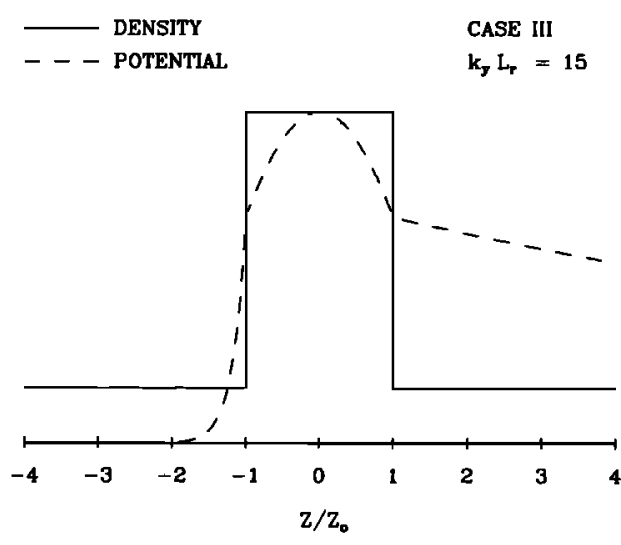

(a)

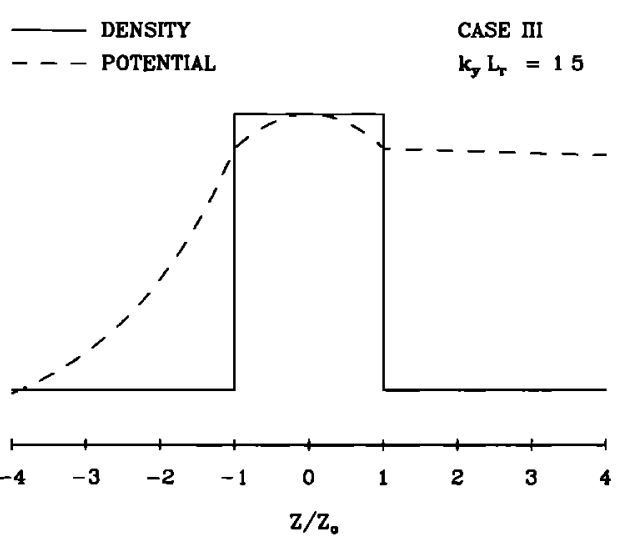

(b)

Fig. 5. Plot of density (solid line) and potential (dashed line) profiles as a function of blob length $\left(z_{0} / z_{r}\right)$. The parameters used are for case III: $(a) k_{y} L_{r}=14.5 ;(b) k_{y} L_{r}=1.45$.

with respect to the interchange mode presented in the previous sections demonstrates that as in the case of the plasma clouds, the finite parallel blob length results in an overall reduction of the growth rate of the interchange instability from the infinite blob case value. This reduction is proportional to the wavelength of the mode: the longer the wavelength, the larger the reduction in its growth rate. In addition, the effects of ion inertia (important at high ionospheric altitudes) are found to reduce the interchange instability growth rate somewhat. The consideration of finite parallel blob length implied that the effects of finite $k_{\|}$on the interchange instability be considered. The parallel extent of the modes is proportional to the transverse wavelength in the ionosphere, and this relationship is roughly given by the relation $\left(L_{\|} / L_{\perp}\right) \propto$ $\left(\sigma_{\|} / \sigma_{\perp}\right)^{1 / 2}$, where $L$ is the characteristic scale size, $\sigma$ is the ionospheric conductivity, and $\perp$ and $\|$ represent components perpendicular and parallel to the magnetic field, respectively. Clearly, as one goes higher in altitude, $\sigma_{\perp}$ (which is proportional to $v_{i n}$ ) decreases, and $\sigma_{\|}$(which is proportional to $v_{e i}{ }^{-1}$ ) increases. Thus with increasing altitude a given transverse scale size mode extends to larger and larger parallel distances. This fact explains the more pronounced reduction of interchange instability at longer transverse wavelengths for the finite-length blob situation. As the mode extends out of the blob (the instability region) into the ambient ionosphere, it attempts to move plasma electrodynamically in these regions (where the density is uniform and this represents a drag for the system). As a result, the growth rate of the interchange process is reduced. For longer transverse wavelengths the parallel extent of the mode is also larger, and thus the "load" also higher for the circuit, and therefore the growth rate reduction is more. Another consequence of the relationship $\left(L_{\|} / L_{\perp}\right) \propto$ $\left(\sigma_{\|} / \sigma_{\perp}\right)^{1 / 2}$ is the fact that the smaller and intermediate scale sizes are localized to shorter parallel distances around the blob (the instability region). Hence, depending on the altitude where the blob is located, the localization may be small enough so that the mode does not extend to the $E$ region on the bottomside. This would mean that the $E$ region would have little impact on the development of the structuring process in the high-latitude $F$ region, whether or not the $E$ region is conducting. The satellite signal scintillations are generally believed to be caused by the intermediate scale sizes (a few hundred meters to a few kilometers), and for a set of typical parameters we have found in our numerical results that this may indeed be the case (see Figures $3 a, 3 b, 4 a$, and $5 a$ ).

The long parallel extension of modes on the topside also means that the regions of low neutral density (and therefore low $v_{i n}$ ) are being traversed by these modes. Thus ion-inertial effects need to be included, and this has an effect of reducing the interchange instability growth rate [Ossakow et al., 1978]. This reduction is also wavelength dependent; the larger transverse wavelengths experience higher reductions in the interchange growth rates (see Figures 1 and 2, curves $B$ and $C$, and also the nonlocal growth rate expressions (B5) and (B6)). However, the interchange process may proceed rather unimpeded at short and intermediate scales, with the reductions in the growth rates less than an order of magnitude even at higher $(\sim 450 \mathrm{~km})$ altitudes, a result in agreement with Ossakow et al. [1978] (see Figures 1 and 2).

Thus we find that the interchange process associated with the high-latitude finite-length blobs is severely hampered for long wavelengths $(\gtrsim 10 \mathrm{~km})$ due to finite blob size, possible coupling to a conducting $E$ region on the bottomside, and inertial effects as modes couple to higher altitudes on the topside. On the other hand, the small and intermediate scale sizes ( $\$ 1 \mathrm{~km}$ and $1-10 \mathrm{~km}$, respectively) may still experience the process of interchange unhindered for the observed blob lengths $(\sim 300 \mathrm{~km})$ for the $F$ region altitudes (i.e., at altitudes around $400-500 \mathrm{~km}$ ). There is evidence that the high-latitude blobs may be displaying such structures [Rino et al., 1983; Cerisier et al., 1985; Tsunoda et al., 1985]. Therefore we suggest that the observed structure in this range $(1-10 \mathrm{~km})$ may be due to the $\mathbf{E} \times \mathbf{B}$ interchange instability caused by the ambient electric fields, or neutral winds, or a combination of both. We have not considered the possible origins of the largescale length structures (blobs, patches, etc.) observed at high latitudes in this work (scales sizes $\gtrsim 100 \mathrm{~km}$ ); on the basis of this work, these are produced by some other mechanism than the interchange process. We have also not attempted to include the dynamics of these large structures or their realistic profiles. The interchange process discussed here develops over a time scale of minutes $\left(\sim 10^{2} \mathrm{~s}\right)$ whereas the long scale size variations occur over a period of hours, thus making the assumption of a "static" blob valid to lowest order. 
In conclusion, we have argued that for the finite-length blobs in the high-latitude $F$ region the intermediate scale size structure responsible for satellite scintillations may be caused by the $\mathbf{E} \times \mathbf{B}$ interchange instability, even in the presence of a conducting $E$ region (for the blob sizes of $\sim 300 \mathrm{~km}$ ). We have also shown that for these scale sizes $(1-10 \mathrm{~km})$ the effect of ion inertia introduces modest reductions in the interchange instability growth rates. All these effects (finite blob length, effect of inertia, etc.), however, act to reduce the growth rate of the interchange instability at long scales ( $\gg 10 \mathrm{~km}$ ) severely, thus making it unlikely that the large scale sizes would be locally produced by the interchange process in the high-latitude $F$ region.

\section{APPENDix A: Local THEORY}

We Fourier analyze the perturbed quantities in the $z$ direction, $f(z) \sim f \exp \left(i k_{z} z\right)$, for the local approximation. Then (23) and (24) result in the dispersion relation

$$
\left[\gamma+{k_{y}}^{2} D_{r}\right]\left[\gamma\left(1+\frac{\gamma}{v_{i n}}\right)-\gamma_{0}\right] \simeq-\left(\gamma \alpha D_{r}\right) k_{z}{ }^{2}
$$

The effects of finite parallel wavelength are included on the right-hand side in the above. We first discuss the case $k_{z}=0$ and later consider the case when $k_{z} \neq 0$.

$k_{z}=0$

Setting $k_{2}=0$ in (A1) leads to the dispersion relation

$$
\left[\gamma+k_{y}^{2} D_{r}\right]\left[\gamma\left(1+\frac{\gamma}{v_{i n}}\right)-\gamma_{0}\right]=0
$$

This equation has a solution for a damped hydromagnetic mode, $\gamma \simeq-k_{y}{ }^{2} D_{r}$ [Chu et al., 1978], and the other solution refers to the $\mathbf{E} \times \mathbf{B}$ instability including the ion-inertial effects [Ossakow et al., 1978]

$$
\gamma\left(1+\frac{\gamma}{v_{i n}}\right)-\gamma_{0}=0
$$

The two limits discussed by Ossakow et al. [1978] are the collisional and inertial limits for the solution of the quadratic in (A3). In the collisional limit, $v_{i n} \gg 4 \gamma_{0}$, one obtains the wellknown result of local theory growth rate of the $\mathbf{E} \times \mathbf{B}$ instability for barium clouds,

$$
\gamma \simeq \gamma_{0}
$$

In the inertial limit, the growth rate of the $\mathbf{E} \times \mathbf{B}$ instabilty is reduced from the collisional case, though not directly proportional to a decrease in $v_{\text {in }}$ (as might be expected at first), but proportional to $\left(v_{i n}\right)^{1 / 2}$ instead [Ossakow et al., 1978]. Thus for $v_{t n}<4 \gamma_{0}$ the growth rate is

$$
\gamma \simeq\left(\gamma_{0} v_{i n}\right)^{1 / 2}
$$

$$
k_{z} \neq 0
$$

We discuss the collisional and inertial limits in the finite $k_{z}$ approximation for the electrostatic and electromagnetic regimes.

Electrostatic regime $\left(k_{y}{ }^{2} D_{r} \gg \gamma\right)$. In an electrostatic ap- proximation the dispersion relation (A1) reduces to

$$
\gamma^{2}+\gamma v_{i n}\left(1+\alpha \frac{k_{z}^{2}}{k_{y}^{2}}\right)-\gamma_{0} v_{\text {in }}=0
$$

The collisional limit corresponds to $4 \gamma_{0} \ll v_{i n}\left(1+\alpha k_{z}{ }^{2} / k_{y}{ }^{2}\right)$, and the growth rate is

$$
\gamma \simeq \gamma_{o} /\left(1+\alpha \frac{k_{z}^{2}}{k_{y}^{2}}\right)
$$

Clearly, the parallel dissipative electron motion has an overall effect of reducing the growth rate in this limit.

The inertial limit is $4 \gamma_{0} \gg v_{i n}\left(1+\alpha k_{z}{ }^{2} / k_{y}{ }^{2}\right)$, and the growth rate in this case is

$$
\gamma \simeq\left(\gamma_{0} v_{i n}\right)^{1 / 2}-\frac{v_{i n}}{2}\left(1+\alpha \frac{k_{z}^{2}}{k_{y}^{2}}\right)
$$

Electromagnetic regime $\left(\gamma \gg k_{y}^{2} D_{r}\right)$. In this case, because of inductive effects, the mode couples to a parallel propagating Alfvén wave which has a stabilizing influence on the instability. The dispersion relation is now

$$
\gamma^{2}+v_{i n} \gamma-\left(\gamma_{0} v_{i n} V_{A}{ }^{2} k_{z}^{2}\right) \simeq 0
$$

The growth rate in the collisional limit is

$$
\gamma \simeq \gamma_{0}-V_{A}^{2} k_{z}^{2} / v_{i n}
$$

This expression is also given by Sperling et al. [1984] and by Basu and Coppi [1983]. In the inertial regime the growth rate is

$$
\gamma \simeq\left(\gamma_{0} v_{\text {in }}-V_{A}{ }^{2} k_{z}{ }^{2}\right)^{1 / 2}
$$

where $V_{A}=\left(B_{0}{ }^{2} / 4 \pi m_{i} n_{0}\right)^{1 / 2}$ is the Alfven speed in the above.

Finite temperature effects. Inclusion of finite temperature effects (i.e., $T \neq 0$ ) results in an additional stabilizing term. The growth rate in the electrostatic limit is given by [see Drake et al., 1985, equation (36)]

$$
\gamma=\left(\gamma_{0}-k_{z}^{2} T / m_{e} v_{e i}\right) /\left(1+\alpha k_{z}^{2} / k_{y}^{2}\right)
$$

If we take $k_{2} \sim \pi / 4 z_{0}$ (see section 3), $z_{0}=150 \mathrm{~km}, T=$ $T_{e}+T_{i}=0.2 \mathrm{eV}$, and $v_{e i}=10^{4} \mathrm{~s}^{-1}$, we find that $k_{z}{ }^{2} T / m_{e} v_{e i}$ $\sim 2 \times 10^{-4} \mathrm{~s}^{-1} \ll \gamma_{0} \sim 10^{-2} \mathrm{~s}$. Thus finite temperature effects are unimportant for the parameters we consider. However, for sufficiently short wavelength modes it can be shown that finite temperature effects stabilize the mode [Drake et al., 1985].

\section{APPENDIX B: NONLOCAL THEORY}

We can rewrite the nonlocal dispersion relation (31) in terms of the normalized parameters as

$$
\begin{aligned}
\tan 2 k_{i} z_{0}= & \left(\frac{1-\hat{\gamma}\left(\hat{v}_{i n} / v_{i n}\right)}{\hat{\gamma} \alpha}\right)_{i}^{1 / 2}\left[\left(\frac{\hat{v}_{i n}}{v_{i n} \alpha}\right)_{1}^{1 / 2}+\left(\frac{\hat{v}_{i n}}{v_{i n} \alpha}\right)_{2}^{1 / 2}\right] \\
& \cdot\left[\left(\frac{1-\hat{\gamma} \hat{v}_{i n} / v_{i n}}{\hat{\gamma} \alpha}\right)_{i}-\left(\frac{\hat{v}_{i n}}{\alpha v_{i n}}\right)_{1}^{1 / 2}\left(\frac{\hat{v}_{i n}}{\alpha v_{i n}}\right)_{2}^{1 / 2}\right]^{-1}
\end{aligned}
$$

where $\hat{v}_{\text {in }}=v_{\text {in }}+\gamma$.

For finite-sized blobs and at higher altitudes the reduced growth rate implies $\hat{\gamma} \lesssim 1$, and in this limit, one may take tan 
$2 k_{i} z_{0} \simeq 2 k_{i} z_{0}$. Then the dispersion relation becomes

$$
\begin{array}{r}
2 \hat{z}_{0}\left(\hat{\gamma}+\hat{k}_{y}{ }^{2}\right)^{1 / 2}=\left\{\hat{\gamma}\left[\left(a_{1}\right)^{1 / 2}+\left(1+\frac{\hat{\gamma}}{v_{i n 2}}\right)^{1 / 2}\left(a_{2}\right)^{1 / 2}\right]\right\} \\
\cdot\left\{1-\hat{\gamma}\left[\left(1+\frac{\hat{\gamma}}{\bar{v}_{t n i}}\right)+\left(a_{1} a_{2}\right)^{1 / 2}\left(1+\frac{\hat{\gamma}}{\bar{v}_{i n 2}}\right)^{1 / 2}\right]\right\}^{-1}
\end{array}
$$

where $a_{n}=\alpha_{1} / \alpha_{n}, \alpha_{n}=\Omega_{e} \Omega_{i} / v_{e i}{ }^{(n)} v_{i n}{ }^{(n)}$, and $\bar{v}_{i n}=v_{i n} / \gamma_{0}$. In the above we have ignored the ion-inertial effects for the bottomside region (1) but retained them for the blob and the topside region (2). Now we write down analytical expressions for the growth rate when $\hat{z}_{0}$ is small.

\section{Case I}

When both the topside and bottomside regions and the blob are collisional, the growth rate is

$$
\hat{\gamma} \simeq \frac{2 \hat{z}_{0} \hat{k}_{y}}{\left[\left(a_{1}\right)^{1 / 2}+\left(a_{2}\right)^{1 / 2}\right]}
$$

where $a_{1}$ and $a_{2}$ represent the fact that we have considered different collisionalities for all three regions. If one considers the situation in which both the topside and bottomside regions may be approximated to be the same, then one obtains the result presented by Sperling et al. [1984]:

$$
\hat{\gamma} \simeq\left(\hat{z}_{0} \hat{k}_{y}\right) / \bar{a}^{1 / 2}
$$

\section{Case II}

In this case, the bottomside and the blob are assumed collisional, but the topside is considered inertial $\left(v_{i n 2}<\gamma_{0}\right)$; then the growth rate is given as

$$
\hat{\gamma} \simeq \frac{2 \hat{z}_{0} k_{y}}{\left[\left(a_{1}\right)^{1 / 2}+\left(a_{2} / \bar{v}_{i n 2}\right)^{1 / 2}\right]}
$$

where $\bar{v}_{i n 2}=v_{i n 2} / \gamma_{0}<1$.

\section{Case III}

In this case, the bottomside is considered collisional, but the ion-inertial effects are included for both the topside region and the blob. Then the growth rate is

$$
\hat{\gamma} \simeq\left(\hat{z}_{0} k_{y}\right) /\left\{\left(a_{1}\right)^{1 / 2}+\left(\frac{a_{2} \hat{\gamma}_{1}}{\bar{v}_{\text {in } 2}}\right)^{1 / 2} / 2\right\}
$$

where

$$
\hat{\gamma}_{1}=\left(\bar{v}_{i n}\right)^{1 / 2},\left[\gamma_{1} \simeq\left(\gamma_{0} v_{i n_{i}}\right)^{1 / 2}\right]
$$

Acknowledgments. This research has been supported by the Defense Nuclear Agency and the Office of Naval Research.

The Editor thanks J. C. Cerisier and J. F. Vickrey for their assistance in evaluating this paper.

\section{REFERENCES}

Basu, B., and B. Coppi, localized plasma depletion in the ionosphere and the equatorial spread F, Geophys. Res. Lett., 10, 900, 1983.
Basu, S., S. Basu, E. Mackenzie, W. R. Coley, W. B. Hanson, and C. $S$. Lin, $F$ region electron density irregularity spectra near auroral acceleration and shear regions, J. Geophys. Res., 89, 5554, 1984.

Cerisier, J. C., J. J. Berthelier, and C. Beghin, Unstable density gradients in the high-latitude ionosphere, Radio Sci., 20, 755, 1985.

Chaturvedi, P. K., and S. L. Ossakow, Nonlinear stabilization of the current convective instability in the diffuse aurora, Geophys. Res. Lett., 6, 957, 1979.

Chaturvedi, P. K. and S. L. Ossakow, The current convective instability as applied to the auroral ionosphere, J. Geophys. Res., 86, 4811, 1981.

Chu, C., M. S. Chu, and T. Ohkawa, Magnetostatic mode and crossfield transport, Phys. Rev. Lett., 4I, 653, 1978.

Drake, J. F., and J. D. Huba, Convective stabilization of ionospheric plasma clouds, J. Geophys. Res., 91, 10,108, 1986.

Drake, J. F., J. D. Huba, and S. T. Zalesak, Finite temperature stabilization of the gradient drift instability in barium clouds, J. Geophys. Res., 90, 5227, 1985.

Farley, D. T., Jr., A theory of electrostatic fields in a horizontally stratified ionosphere subject to a vertical magnetic field, J. Geophys. Res., 64, 1225, 1959.

Fejer, B. G., and M. C. Kelley, Ionospheric irregularities, Rev. Geophys., $18,401,1980$.

Fremouw, E. J., C. L. Rino, R. C. Livingston, and M. C. Cousins, A persistent subauroral scintillation enhancement observed in Alaska, Geophys. Res. Lett., 4, 539, 1977.

Goldman, S. R., L. Baker, S. L. Ossakow, and A. J. Scannapieco, Striation formation associated with barium clouds in an inhomogeneous ionosphere, J. Geophys. Res., 81, 5097, 1976.

Huba, J. D., and P. K. Chaturvedi, The effect of finite "blob" size on the current convective instability in the auroral ionosphere, J. Geophys. Res., 91, 7125, 1986.

Huba, J. D., and S. L. Ossakow, Influence of magnetic shear on the current convective instability in the diffuse aurora, $J$. Geophys. Res., $85,6874,1980$.

Kelley, M. C., J. F. Vickrey, C. W. Carlson, and R. Torbert, On the origin and spatial extent of high-latitude $F$ region irregularities, $J$. Geophys. Res., 87, 4469, 1982.

Kelly, J. D., and J. F. Vickrey, $F$-region ionospheric structure associated with antisunward flow near the dayside polar cusp, Geophys. Res. Lett., 11(9), 907, 1984.

Keskinen, M. J., and S. L. Ossakow, Nonlinear evolution of plasma enhancements in the auroral ionosphere, 1, Long wavelength irregularities, J. Geophys. Res., 87, 144, 1982.

Keskinen, M. J., and S. L. Ossakow, Nonlinear evolution of convecting plasma enhancements in the auroral ionosphere, 2, Small scale irregularities, J. Geophys. Res., 88, 474, 1983.

Mitchell, H. G., Jr., J. A. Fedder, M. J. Keskinen, and S. T. Zalesak, A simulation of high-latitude $F$-layer instabilities in the presence of magnetosphere-ionosphere coupling, Geophys. Res. Lett., 12, 283, 1985.

Ossakow, S. L., and P. K. Chaturvedi, Current convective instability in the diffuse aurora, Geophys. Res. Lett., 6, 332, 1979.

Ossakow, S. L., P. K. Chaturvedi, and J. B. Workman, High altitude limit of the gradient drift instability, J. Geophys. Res., 83, 2691, 1978.

Rino, C. L., R. C. Livingston, and S. J. Mathews, Evidence for sheetlike auroral ionospheric irregularities, Geophys. Res. Lett., 5, 1039, 1978.

Rino, C. L., R. C. Livingston, R. T. Tsunoda, R. M. Robinson, J. F. Vickrey, C. Senior, M. D. Cousins, J. Owen, and J. A. Klobuchar, Recent studies of the structure and morphology of auroral zone $F$ region irregularities, Radio $S$ ci., $18,1167,1983$.

Scannapieco, A. J., S. R. Goldman, S. L. Ossakow, D. L. Book, and B. E. McDonald, Theoretical and numerical simulation studies of mid-latitude $F$ region irregularities, $N R L$ Memo. Rep., 3014, 1975.

Sojka, J. J., and R. W. Schunk, A theoretical study of the production and decay of localized electron density enhancements in the polar ionosphere, J. Geophys. Res., 91, 3245, 1986.

Sperling, J. L., J. F. Drake, S. T. Zalesak, and J. D. Huba, The role of finite parallel length on the stability of barium clouds, J. Geophys. Res., 89, 10,913, 1984.

Tsunoda, R. T., I. Häggström, A. Pellinen-Wannberg, Å. Steen, and $G$. Wannberg, Direct evidence of plasma density structuring in the auroral $F$ region ionosphere, Radio $S c i ., 20,762,1985$.

Vickrey, J. F., C. L. Rino, and T. A. Potemra, Chatanika/TRIAD 
observations of unstable ionization enhancements in the auroral F-region, Geophys. Res. Lett., 7(10), 789, 1980.

Völk, H. J., and G. Haerendel, Striations in ionospheric ion clouds, 1, J. Geophys. Res., 76, 4541, 1971.

Weber, E. J., J. Buchau, J. G. Moore, J. R. Sharber, R. C. Livingston, J. D. Winningham, and B. W. Reinisch, $F$ layer ionization patches in the polar cap, J. Geophys. Res., 89, 1683, 1984.
P. K. Chaturvedi and J. D. Huba, Geophysical and Plasma Dynamics Branch, Plasma Physics Division, Naval Research Laboratory, Washington, DC 20375.

(Received September 29, 1986;

revised December 11, 1986;

accepted December 18, 1986.) 